\title{
The Effect of a Grain Boundary on Deformation in an Aluminum Bicrystal with a Common Tensile Axis of [100]
}

\author{
Keizo Kashihara $^{1, *}$ and John A. Wert ${ }^{2}$ \\ ${ }^{1}$ Wakayama National College of Technology, Gobo 644-0023, Japan \\ ${ }^{2}$ Materials Research Department, Ris $\phi$ National Laboratory, Roskilde 4000, Denmark
}

\begin{abstract}
The effect of a grain boundary on deformation has been examined using an aluminum bicrystal specimen composed of crystals having a common tensile axis of [100] by the measurement of orientation change with the electron backscatter diffraction technique. After deformation to a strain of $20 \%$, the crystal rotation axis (CRA) map, which provides the orientation distribution of crystal rotation axes relative to the initial crystal orientations, reveals areas affected by the grain boundary. The thickness of the affected zone was $100 \mu \mathrm{m}$ in one crystal and $150 \mu \mathrm{m}$ in the other. In both crystals, the crystal rotation axis relative to the initial crystal orientation differed between the affected zone and the crystal interior. In the crystal interior, two slip systems showed a higher activity than the other slip systems, whereas in the affected zone, three or four slip systems were more active than the other slip systems. The CRA map showed that the width and shape of the affected zones across the grain boundary were not symmetrical with respect to the grain boundary.
\end{abstract}

(Received September 30, 2005; Accepted December 9, 2005; Published January 15, 2006)

Keywords: aluminum, bicrystal, deformation, crystal orientation, slip system, electron backscatter diffraction (EBSD)

\section{Introduction}

It is well known that inhomogeneous deformation in polycrystals, which causes work hardening, occurs inside grains, near grain boundaries (GBs), and around second phase particles. As a fundamental study, deformation in orientation-controlled single crystals, bicrystals, and tricrystals have been studied. For example, slip systems activated near GBs were examined using bicrystals through the stress transmission mechanism ${ }^{1)}$ and the slip transfer mechanism. ${ }^{2,3)}$

The electron backscatter diffraction (EBSD) technique has been employed in determining orientation distribution in, not only polycrystals, ${ }^{4-8)}$ but also single crystals. ${ }^{9-20)}$ The EBSD technique has the advantage that it enables precise and automatic measurement of many crystal orientations over a large area. Wert et al. ${ }^{18)}$ have performed an EBSD analysis to determine active slip systems in aluminum single-crystals deformed in tension along [110]. The orientations of crystal rotation axes calculated from shear strains due to slip systems receiving the maximum resolved shear stress were compared with those obtained by the EBSD analysis. They could elucidate the mechanism of structure development by slip systems activated with different shear strains.

This analysis method has recently been applied to a grain in a copper polycrystal specimen deformed in tension in order to investigate the effect of grain interactions on orientation subdivision. ${ }^{8)}$ Crystal orientation maps, which provide the crystal rotation axes relative to the initial crystal orientations, revealed predominant coarse domains and special domains with different features. The interactions between the grain and the neighboring grains were remarkable in the special domains, which included layer-shaped patterns in orientation along boundary segments, lobe-shaped patterns in the vicinity of triple grain junctions and a plate-shaped pattern extending from near a triple grain junction.

*Corresponding author, E-mail: kashihara@wakayama-nct.ac.jp
Several EBSD studies have been conducted using aluminum bicrystals deformed in $\operatorname{cold}^{21)}$ or hot plane strain compression $^{22)}$ and niobium bicrystals in cold rolling. ${ }^{23)}$ In the present study, we examine the effect of GB on deformation in an aluminum bicrystal specimen having a common tensile axis of [100]. In particular, the number and type of slip systems necessary for the deformation are discussed on the basis of the geometrical crystal rotation axes of active slip systems and the EBSD analysis originally performed in the single-crystal experiment. ${ }^{18}$ )

\section{Experimental Procedure}

A bicrystal specimen was prepared from a 99.99 mass $\%$ purity ingot by a modified Bridgman method. Figure 1 schematizes the specimen. The left and right component crystals are called Crystal A and Crystal B, respectively. The grain boundary (GB) between them is a $45^{\circ}$ asymmetric tilt boundary parallel to the tensile axis (TA) and the front face normal (FF). The common tensile axis of the component crystals is [100]. The tensile axis orientation of each crystal is within $1^{\circ}$ of the ideal orientation. The test part of the specimen is $20.0 \mathrm{~mm}$ in length, $9.2 \mathrm{~mm}$ in width, and $4.0 \mathrm{~mm}$ in thickness. After electro-polishing to obtain a mirror surface, the specimen was deformed in tension to $20 \%$ at room temperature. The slip trace pattern on the front face was observed using a JEOL 6400 scanning electron microscope (SEM). The EBSD measurement was carried out using a JEOL 840 SEM equipped with an automated indexing system developed by Krieger Lassen et al. ${ }^{24)}$ The EBSD patterns were taken at 2 and $20 \mu \mathrm{m}$ intervals on FF. The patterns detected were converted into orientations of crystal rotation axes and crystal rotation angles relative to the initial crystal orientations.

Table 1 lists twelve slip systems with the orientation of crystal rotation axes and the Schmid factor for [100] extension. The notation of slip systems follows the standard Bishop-Hill notation. ${ }^{25}$ ) We have chosen the crystal rotation 


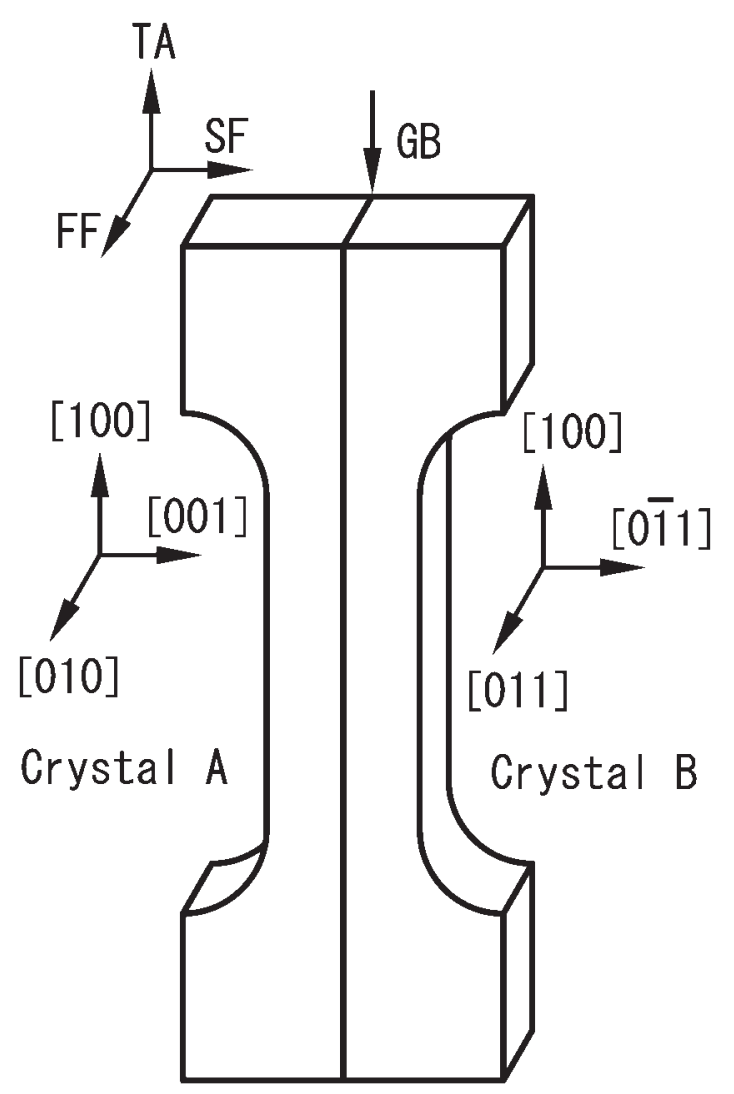

Fig. 1 Schematic illustration of pure aluminum bicrystal specimen for tensile testing. Component crystals are related through a $45^{\circ}$ rotation around a common tensile axis of [100].

Table 1 Twelve slip systems, the orientation of crystal rotation axes and the Schmid factor for [100] extension.

\begin{tabular}{rcccc}
\hline $\begin{array}{c}\text { Active slip } \\
\text { system }\end{array}$ & Slip direction & Slip plane & $\begin{array}{c}\text { Crystal } \\
\text { rotation axis }\end{array}$ & Schmid factor \\
\hline $\mathrm{a} 1$ & {$[01 \overline{1}]$} & $(11 \overline{1})$ & {$[2 \overline{1} \overline{1}]$} & zero \\
$-\mathrm{a} 2$ & {$[10 \overline{1}]$} & $(11 \overline{1})$ & {$[1 \overline{2} 1]$} & non-zero \\
$\mathrm{a} 3$ & {$[1 \overline{1} 0]$} & $(11 \overline{1})$ & {$[\overline{1} \overline{1} 2]$} & non-zero \\
$\mathrm{b} 1$ & {$[011]$} & $(11 \overline{1})$ & {$[\overline{2} 1 \overline{1}]$} & zero \\
$-\mathrm{b} 2$ & {$[101]$} & $(11 \overline{1})$ & {$[\overline{1} 21]$} & non-zero \\
$\mathrm{b} 3$ & {$[1 \overline{1} 0]$} & $(11 \overline{1})$ & {$[112]$} & non-zero \\
$-\mathrm{c} 1$ & {$[01 \overline{1}]$} & $(1 \overline{1} \overline{1})$ & {$[\overline{2} \overline{1} \overline{1}]$} & zero \\
$-\mathrm{c} 2$ & {$[101]$} & $(1 \overline{1} \overline{1})$ & {$[12 \overline{1}]$} & non-zero \\
$\mathrm{c} 3$ & {$[110]$} & $(1 \overline{1} \overline{1})$ & {$[\overline{1} \overline{1} \overline{2}]$} & non-zero \\
$-\mathrm{d} 1$ & {$[01 \overline{1}]$} & $(1 \overline{1} 1)$ & {$[21 \overline{1}]$} & zero \\
$-\mathrm{d} 2$ & {$[10 \overline{1}]$} & $(1 \overline{1} 1)$ & {$[\overline{1} \overline{2} \overline{1}]$} & non-zero \\
$\mathrm{d} 3$ & {$[110]$} & $(1 \overline{1} 1)$ & {$[1 \overline{1} \overline{2}]$} & non-zero \\
\hline
\end{tabular}

axis for each slip system that gives a positive rotation in the right hand system. In Crystals A and B with a tensile axis of [100], eight slip systems (-a2, a3, -b2, b3, -c2, c3, -d2 and $\mathrm{d} 3$ ) with non-zero in the Schmid factor are activated.

\section{Results}

\subsection{Slip trace observation}

Figure 2 shows slip trace patterns in Crystals A and B across GB. Both crystals reveal multiple slips. Crystal A exhibits slips on (111) and (111) (denoted as $(111) /(1 \overline{1} 1))$

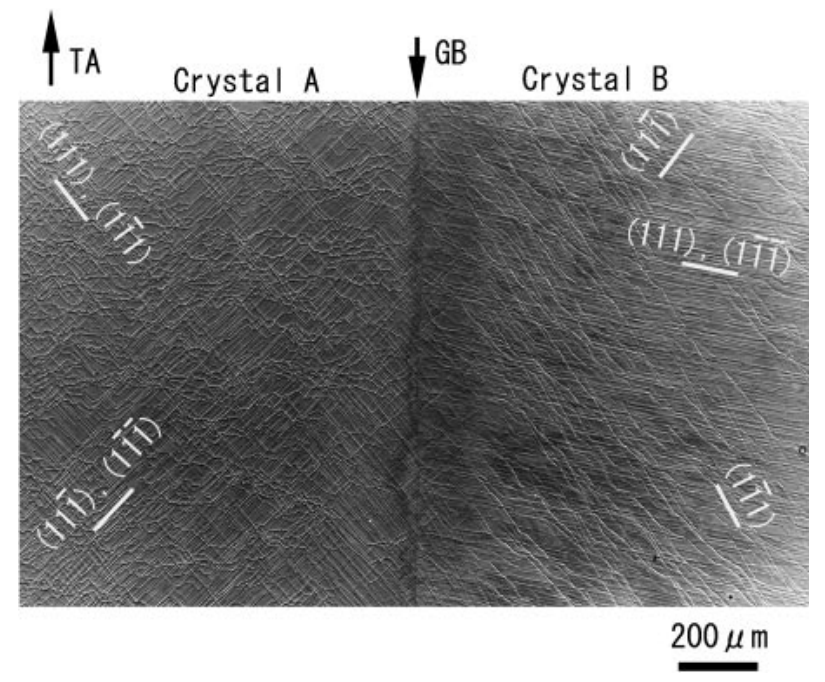

Fig. 2 SEM micrograph showing slip patterns on the front face after deformation to $20 \%$. White lines indicate the slip traces.

and slips on $(11 \overline{1}) /(1 \overline{1} \overline{1})$. Cross slips between $(111) /(1 \overline{1} 1)$ and $(1 \overline{1} \overline{1}) /(1 \overline{1} \overline{1})$ can be identified. Cross slips with large steps mainly occur in the crystal interior, whereas cross slips with fine steps are found in the vicinity of GB. Crystal B reveals slips on $(11 \overline{1}),(1 \overline{1} 1)$ and $(111) /(1 \overline{1} \overline{1})$, between which cross slips are observed. We can distinguish an area along GB that has an image contrast different from the crystal interior. This area is about $140 \mu \mathrm{m}$ in width and is presumably caused by GB. We shall call this area under the effect of GB the affected zone. The slip trace patterns do not show any formation of deformation bands.

\subsection{EBSD analysis}

Figure 3(a) shows an orientation image map measured at $20 \mu \mathrm{m}$ intervals. The map consisting of 40,000 data points provides orientations of crystal rotation axes relative to the initial crystal orientations. We shall call this map the crystal rotation axis (CRA) map; it follows the way of mapping in the previous study. ${ }^{18)}$ The CRA map refers to a standard pole figure in Fig. 3(b), color-coded for orientations of crystal rotation axes. If a data point rotates around an axis of TA relative to the initial crystal orientation, the point is colored red in Fig. 3(a). Data points colored green and blue represent crystal rotations around the axes of FF and the side face normal (SF), respectively. In Fig. 3(a), the interiors of Crystals $\mathrm{A}$ and $\mathrm{B}$ are colored blue and green, which refer to crystal rotations around the axes near $\pm \mathrm{SF}$ and $\pm \mathrm{FF}$, respectively. The vicinity of GB is red, dark orange and light green, and so on. The colors indicate that the vicinity of GB exhibits various crystal rotation axes different from the crystal interiors. Thus, GB influences the crystal rotation in its vicinity, so that the formation of the affected zones across GB is attributed to GB. Although the component crystals were deformed in tension along the same [100] axis, the crystal interiors exhibit different crystal rotation axes.

Figure 3(c) plots orientations of crystal rotation axes and crystal rotation angles relative to the initial crystal orientations along the line $\mathrm{V}-\mathrm{V}^{\prime}$ in Fig. 3(a). The interiors of Crystal A (blue) and Crystal B (green) rotate by $3^{\circ}-6^{\circ}$ 
(a)

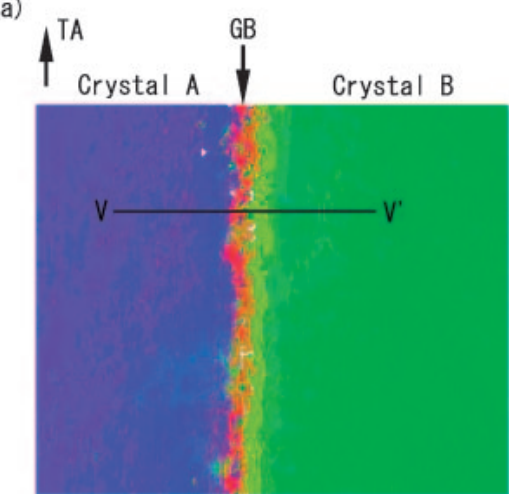

(c)

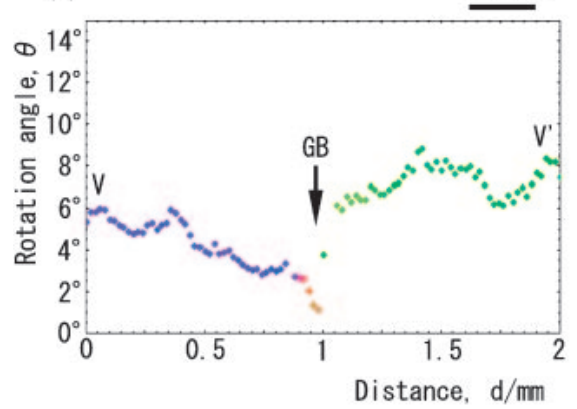

Fig. 3 (a) A crystal rotation axis (CRA) map measured at $20 \mu \mathrm{m}$ intervals. (b) A reference pole figure showing the color coding for orientations of crystal rotation axes described in Ref. 18. The crystal rotations around $\pm \mathrm{TA}, \pm \mathrm{FF}$ and $\pm \mathrm{SF}$ are represented with red, green, and blue, respectively. (c) A plot graph of crystal rotation axes and angles across the GB.

around $\pm \mathrm{SF}$ and by $6^{\circ}-9^{\circ}$ around $\pm \mathrm{FF}$, respectively. On average, Crystal A has a smaller crystal rotation angle than Crystal B. In both crystals, the rotation angle decreases as GB is approached.

Figures 4(a) and (b) show a CRA map measured at $2 \mu \mathrm{m}$ intervals and the standard pole figure. The CRA map shows that Crystals A and B form affected zones about 100 and $150 \mu \mathrm{m}$ in width, respectively. Crystal A is blue outside the affected zone, and green and light green inside. The area adjoining GB shows a variety of colors such as blue, purple, red and dark orange. The change of color indicates that the orientations of crystal rotation axes shift from the axes near \pm SF (blue) to those near \pm FF (green) as one approaches GB. The area adjoining GB is found to have various crystal rotation axes relative to the initial crystal orientation. The interior of Crystal B is colored green. The affected zone is colored light green and dark orange, depending on the distance from GB. The orientations of crystal rotation axes change from the axes near $\pm \mathrm{FF}$ to the axes near $\langle 111\rangle$. We find that $\langle 111\rangle$ in Crystal $\mathrm{B}$ is located between $\pm \mathrm{FF}$ and $\pm \mathrm{TA}$, as shown in Fig. 5(b). The affected zones of the component crystals are asymmetrical with respect to GB in width and shape. The affected zone of Crystal B is wider than that of Crystal A, so that Crystal B is presumably more affected by GB than Crystal A. The affected zone of Crystal B appears feathery with dark orange.

Figure 4(c) plots orientations of crystal rotation axes and the crystal rotation angles along the line $X-X^{\prime}$ in Fig. 4(a). Crystal A consistently has a smaller rotation angle than
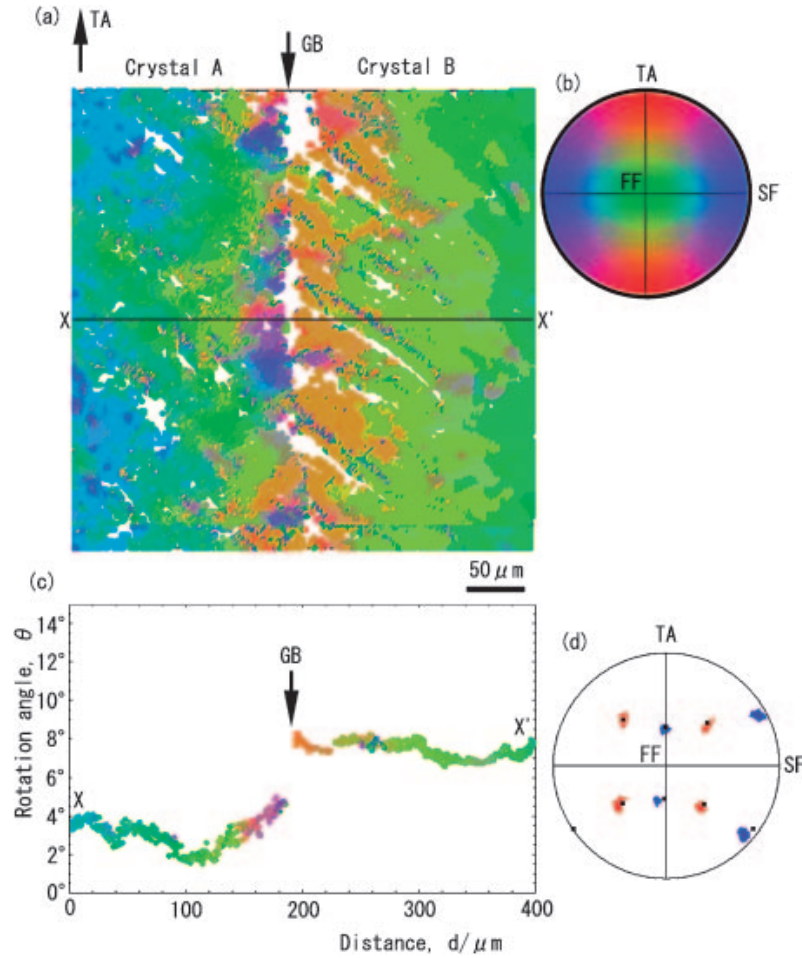

Fig. 4 (a) A CRA map measured at $2 \mu \mathrm{m}$ intervals. (b) The reference pole figure. (c) A plot graph of crystal rotation axes and angles. (d) $\langle 111\rangle$ orientations of Crystal A (red dots) and Crystal B (blue dots) after deformation together with the initial crystal orientation (black squares).

Crystal B. The average of crystal rotation angles is about $3^{\circ}$ in Crystal $\mathrm{A}$ and $7^{\circ}$ in Crystal B. Figure 4(d) shows a $\langle 111\rangle$ pole figure of the crystal orientations after deformation (colored dots) together with the initial crystal orientations (black squares). The red and blue colors correspond to the crystal orientations of Crystals A and B, respectively. The crystal orientation in Crystal A is distributed within $10^{\circ}$ around the initial crystal orientation. The orientation distribution is typical of multiple slip deformation. Crystal B also has crystal orientations distributed within $10^{\circ}$. All of them rotate by about $6^{\circ}$ on average around the $[\overline{1} \overline{1} \overline{1}]$ axis relative to the initial crystal orientation.

\section{Discussion}

\subsection{Active slip systems}

Affected zones of GB were clearly observed to form along GB. We discuss slip systems activated in the affected zones and the crystal interiors. The following two facts should be noted for the analysis of active slip systems in multiple slip deformation.

(1) The eight slip systems receiving the same resolved shear stress (with non-zero in the Schmid factor) can operate from start to finish in deformation. The orientation of the geometrical crystal rotation axis for each slip system is shown in Table 1. The slip pattern observation showed the multiple slips without deformation bands. This implies that these slip systems have been activated in the component crystals.

(2) Non-equivalent slips of the eight slip systems occur when all of them are in operation. Appreciable crystal 
(a)

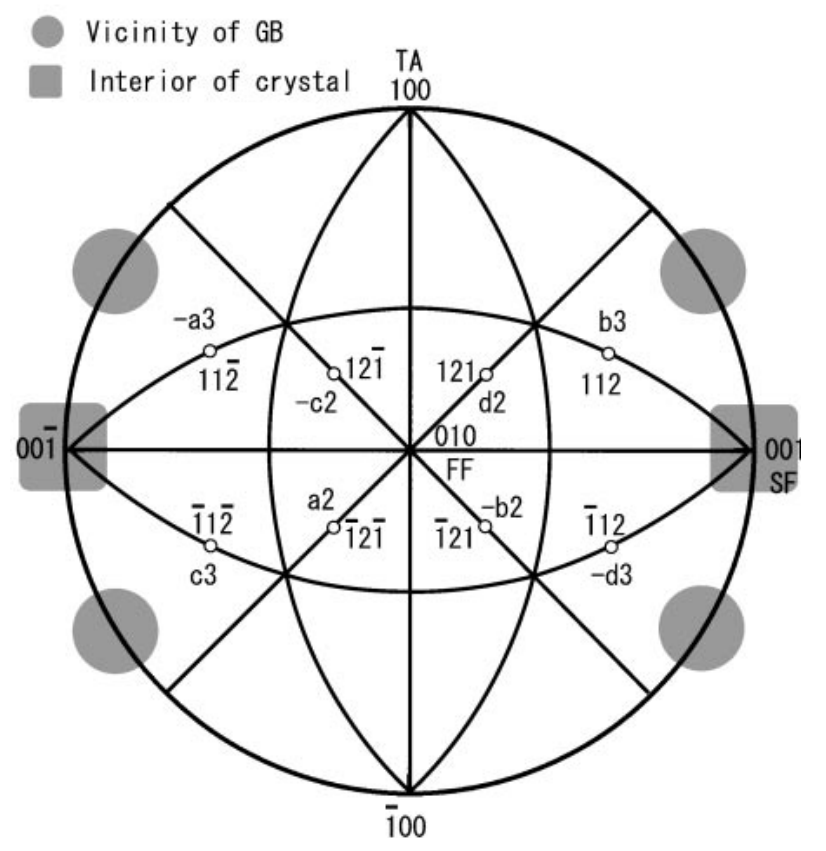

(b)

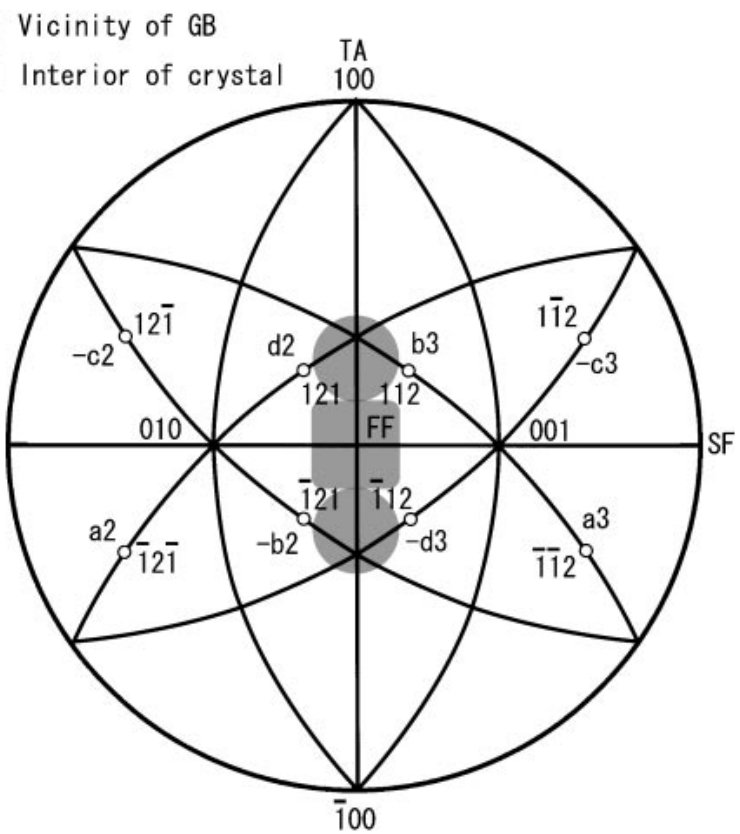

Fig. 5 Pole figures showing orientations of rotation axes by circles for eight active slip systems and orientations of actual crystal rotation axes observed in Crystal A (a) and Crystal B (b) by shaded zones.

rotations relative to the initial crystal orientations were obtained from the CRA maps. The interiors of Crystals $\mathrm{A}$ and $\mathrm{B}$ rotated by a maximum of about $6^{\circ}$ and $9^{\circ}$, respectively. This means that a slip system or several slip systems operate more actively than other slip systems. Non-equivalent slips produce a crystal rotation around a specific crystal rotation axis.

Figure 5 shows orientations of geometrical crystal rotation axes in Crystals A and B. Each open circle represents the orientation of the crystal rotation axis for each active slip
Table 2 Observed orientations of crystal rotation axes and the combination of slip systems geometrically required for the crystal rotations.

\begin{tabular}{|c|c|c|c|}
\hline \multicolumn{4}{|c|}{ Crystal A } \\
\hline \multicolumn{2}{|c|}{ Crystal interior } & \multicolumn{2}{|c|}{ The vicinity of grain boundary } \\
\hline $\begin{array}{l}\text { Rotation } \\
\text { axis }\end{array}$ & $\begin{array}{l}\text { Combination } \\
\text { of slip system }\end{array}$ & $\begin{array}{l}\text { Rotation } \\
\text { axis }\end{array}$ & $\begin{array}{l}\text { Combination } \\
\text { of slip system }\end{array}$ \\
\hline \multirow[t]{2}{*}[001]{} & $-\mathrm{a} 2-\mathrm{b} 2$ & {$[102]$} & $-\mathrm{a} 2+\mathrm{a} 3+\mathrm{b} 3-\mathrm{c} 2$ \\
\hline & $a 3+b 3$ & & $\mathrm{a} 3+\mathrm{b} 3+\mathrm{b} 3+\mathrm{d} 3$ \\
\hline \multirow[t]{6}{*}[00\overline{1}]{} & $-\mathrm{c} 2-\mathrm{d} 2$ & {$[\overline{102}]$} & $\mathrm{a} 3+\mathrm{a} 3+\mathrm{b} 3+\mathrm{c} 3$ \\
\hline & $\mathrm{c} 3+\mathrm{d} 3$ & & $\mathrm{a} 3-\mathrm{b} 2+\mathrm{b} 3-\mathrm{d} 2$ \\
\hline & & {$[10 \overline{2}]$} & $-\mathrm{a} 2-\mathrm{c} 2+\mathrm{c} 3+\mathrm{d} 3$ \\
\hline & & & $\mathrm{b} 3+\mathrm{c} 3+\mathrm{d} 3+\mathrm{d} 3$ \\
\hline & & {$[\overline{1} 0 \overline{2}]$} & $\mathrm{a} 3+\mathrm{c} 3+\mathrm{c} 3+\mathrm{d} 3$ \\
\hline & & & $-\mathrm{b} 2+\mathrm{c} 3-\mathrm{d} 2+\mathrm{d} 3$ \\
\hline \multicolumn{4}{|c|}{ Crystal B } \\
\hline \multicolumn{2}{|c|}{ Crystal interior } & \multicolumn{2}{|c|}{ The vicinity of grain boundary } \\
\hline $\begin{array}{l}\text { Rotation } \\
\text { axis }\end{array}$ & $\begin{array}{l}\text { Combination } \\
\text { of slip system }\end{array}$ & $\begin{array}{l}\text { Rotation } \\
\text { axis }\end{array}$ & $\begin{array}{c}\text { Combination } \\
\text { of slip system }\end{array}$ \\
\hline \multirow[t]{2}{*}[011]{} & $-\mathrm{b} 2+\mathrm{b} 3$ & {$[233]$} & $-a 2-b 2+b 3-c 2$ \\
\hline & $\mathrm{a} 3-\mathrm{c} 2$ & {$[\overline{2} 33]$} & $\mathrm{a} 3-\mathrm{b} 2+\mathrm{b} 3+\mathrm{c} 3$ \\
\hline \multirow[t]{2}{*}[0\overline{1}\overline{1}]{} & $-\mathrm{d} 2+\mathrm{d} 3$ & {$[2 \overline{3} \overline{3}]$} & $-\mathrm{a} 2-\mathrm{c} 2-\mathrm{d} 2+\mathrm{d} 3$ \\
\hline & $-\mathrm{a} 2+\mathrm{c} 3$ & {$[\overline{2} \overline{3} \overline{3}]$} & $\mathrm{a} 3+\mathrm{c} 3-\mathrm{d} 2+\mathrm{d} 3$ \\
\hline
\end{tabular}

system. For example, the operation of the slip system of b3 in Crystal A produces a crystal rotation around [112] relative to the initial crystal orientation. Table 2 shows the combination of slip systems necessary for the actual crystal rotations observed. The combination is calculated to produce a crystal rotation by a minimum number of slip systems, based on the orientation of the geometrical crystal rotation axis for each slip system shown in Table 1. In Fig. 3, the interior of Crystal A is blue, which corresponds to the crystal rotation around an axis near [001] or [001]. The crystal rotation axes are indicated by shadowed squares in Fig. 5(a). An extra activity of the pair of slip systems $-\mathrm{a} 2$ and $-\mathrm{b} 2$ can produce the crystal rotation around the [001] axis. An extra activity of a3 and b3 also generates the crystal rotation around [001]. The rotation around [001] needs an extra activity of $-\mathrm{c} 2$ and $-\mathrm{d} 2$ or $\mathrm{c} 3$ and $\mathrm{d} 3$.

The area adjoining GB inside the affected zone of Crystal A had various orientations of crystal rotation axes. We deal with a purple area adjoining GB on the line $\mathrm{X}-\mathrm{X}^{\prime}$ in Fig. 4. Purple corresponds to the orientations of crystal

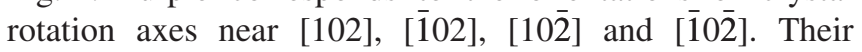
orientations are indicated by shadowed circles in Fig. 5(a). Combined slips by $-\mathrm{a} 2$, a3, b3 and $-\mathrm{c} 2$ can produce the crystal rotation around [102]. Combined slips by a3, b3, b3 and $\mathrm{d} 3$ also generate the crystal rotation around [102]. Here, "double b3" means that b3 has twice the activity of a3 and d3. The slip systems necessary for the crystal rotations around [ $\overline{1} 02],[10 \overline{2}]$ and [ $\overline{1} 0 \overline{2}]$ are also represented in Table 2.

The interior of Crystal B had the orientation of crystal rotation axis near [011] or [0 $\overline{1} \overline{1}]$, which is indicated by a shadowed square in Fig. 5(b). If the two slip systems -b2 and $b 3$ or a3 and $-c 2$ operate more actively than the others, the combined slips produce the crystal rotation around [011]. The crystal rotation around $[0 \overline{1} \overline{1}]$ requires an extra activity by $-\mathrm{d} 2$ and $\mathrm{d} 3$ or $-\mathrm{a} 2$ and $\mathrm{c} 3$. 
The area adjoining GB inside the affected zone of Crystal B was mostly colored dark orange. This corresponds to the orientations of crystal rotation axes near $\langle 111\rangle$ located between \pm FF and \pm TA. We discuss here the crystal rotations around [233], [2 333$],[2 \overline{3} \overline{3}]$ and $[\overline{2} \overline{3} \overline{3}]$, which are represented by shadowed circles in Fig. 5(b). An extra activity by - a2, $-\mathrm{b} 2, \mathrm{~b} 3$ and $-\mathrm{c} 2$ can generate the crystal rotation around [233]. The crystal rotation around [233] results from an extra activity by a3, - b2, b3, and c3. The combinations of slip systems necessary for the other crystal rotations are also listed in Table 2.

The CRA maps revealed the affected zone formation even in the multiple slip deformation by eight slip systems in each component crystal. The number and type of active slip systems have been revealed by a comparison between the CRA map and the crystal rotation axes of the active slip systems. The crystal rotations of the interiors of Crystals A and B are the result of two slip systems operating more actively than the other slip systems. In contrast, the crystal rotations in the affected zones in the vicinity of GB are the result of extra slips of three or four slip systems. Clearly, GB increases the number of slip systems operating more actively than other slip systems and enables the production of arbitrary crystal rotations relative to the initial crystal orientations.

Slips occurring at GBs in bicrystals have been investigated in terms of macroscopic and microscopic compatibility. 1,26,27) One experiment showed that macroscopic continuity at GB would in general require the crystals of a bicrystal to deform on at least four slip systems between them, either two in both crystals or three in one crystal and one in the other. ${ }^{1)}$

Tatschl and Kolednik have recently examined active slip systems in copper polycrystal specimens by combining local crystal orientations and local in-plane strains. ${ }^{7)}$ The crystal orientations were measured by EBSD analyses that were taken before and after a desired deformation step. The local in-plane strains were determined from micrographs, again taken before and after the deformation step. Very strong heterogeneities in local lattice rotation and in-plane strain existed within single grains. At some GBs, very high lattice rotations were observed. More than three independent slip systems had to be activated in some GB regions to accommodate the influence of the neighboring grain, while three slip systems seemed to be enough in most regions of the grain interiors. The result is similar to our result, which indicates that the affected zone in the vicinity of GB needs more than two slip systems (operating more actively than the other slip systems in our case), while the crystal interior requires two slip systems (operating more actively than the other slip systems), although we used a bicrystal specimen consisting of component crystals with the tensile axis of a common [100] and they used a copper polycrystal specimen consisting of arbitrarily oriented grains with an average size of below $100 \mu \mathrm{m}$.

\subsection{Affected zone formation across GB}

The effect of GB on deformation has been derived from local strains in the measurement of the plastic displacement field in an aluminum polycrystal specimen deformed in channel die compression ${ }^{6)}$ and copper polycrystals deformed in tension. ${ }^{28)}$

Zaefferer et $a .^{21)}$ elucidated the influence of GB on deformation up to $30 \%$ strain using aluminum bicrystals with $8.7^{\circ}, 15.4^{\circ}$ and $31.5^{\circ}$ symmetrical $\langle 112\rangle$ tilt GBs. High resolution orientation maps obtained from EBSD patterns were compared with the analyses of a crystal elasticityplasticity finite element model, Sachs model, Taylor and modified Taylor models. The influence of GB on kinematics of deformation was not evident. Zaefferer et al. mentioned that observed differences between the three bicrystals were not due to the changing structure of GBs, but rather to the different orientations of these crystals. However, careful inspection of the high resolution orientation maps revealed the influence of GB on the change of the crystal orientation close to GB, especially in the deformed bicrystal with an originally $15.4^{\circ} \mathrm{GB}$. The crystal rotations relative to the initial crystal orientations appeared to be about $15^{\circ}$ around the transverse direction.

The deformation of the bicrystal specimen having an asymmetric tilt GB in the present study introduced multiple slips by eight active slip systems receiving the same resolved shear stress. Even in the deformation structure having a misorientation within $10^{\circ}$, we could distinguish the affected zones in the CRA maps which provided the orientation distribution of crystal rotation axes relative to the initial crystal orientations. Clearly, the crystal rotation axes in the affected zones differ from those in the crystal interiors. The former rotation is caused by GB. The CRA map developed by Wert et al., which was originally applied to single-crystal deformation ${ }^{18)}$ and recently to polycrystal deformation, ${ }^{8)}$ is found to be an effective way for the identification of the affected zones formed as a result of the effect of GB in the orientation-controlled bicrystal specimen. In addition, the width and shape of the affected zones were not symmetrical with respect to GB. The effect of GB was observed to extend over a distance of $100 \mu \mathrm{m}$ in Crystal A and $150 \mu \mathrm{m}$ in Crystal B. The affected zone of Crystal B showed a feathery appearance. On the basis of the CRA map, Crystal B was influenced more strongly by GB than Crystal A.

\subsection{Crystal rotation axes and crystal rotation angles in crystal interiors}

The bicrystal specimen consisted of Crystal A with [100](010) orientation and Crystal B with [100](011) orientation. Although the component crystals were deformed along the same tensile axis of [100], the CRA maps showed that the crystal interiors had different orientations of crystal rotation axes. Crystal A rotated around the axes near $\pm \mathrm{SF}$ relative to the initial crystal orientation, while Crystal B rotated around the axes near $\pm \mathrm{FF}$ relative to the initial crystal orientation. Crystal B always showed larger rotation angles than Crystal A. The rotation angles of Crystals A and B were $3^{\circ}-6^{\circ}$ and $6^{\circ}-9^{\circ}$, respectively. The differences between the crystals could be explained by the slight deviation of the tensile axes of the two crystals from [100]. However, the deviation was within $1^{\circ}$. The crystal rotations relative to the initial orientations are much larger than the deviation. Consequently, in addition to the effect of the slight deviation of tensile axes, another reason should be considered, although at present the reason is not clear. 


\section{Conclusions}

The aluminum bicrystal specimen with an asymmetric tilt GB consisting of crystals having the common tensile axis of [100] was deformed in tension to $20 \%$ at room temperature. Crystal A with [100](010) and Crystal B with [100](011) show multiple slips without forming deformation bands. The main conclusions obtained in this study are as follows:

(1) The crystal rotation axis (CRA) map reveals areas affected by GB called the affected zones. The thickness of the zone is $100 \mu \mathrm{m}$ in Crystal A and $150 \mu \mathrm{m}$ in Crystal B. The area adjoining GB inside the affected zone of Crystal A rotates around various crystal rotation axes relative to the initial crystal orientations. The area adjoining GB inside the affected zone of Crystal B mostly has crystal rotation axes near $\langle 111\rangle$. The interiors of Crystals A and B had crystal rotation axes near the side face normal and the front face normal, respectively.

(2) The crystal interiors are subjected to the non-equivalent activity of eight slip systems with two slip systems showing a higher activity than the remaining slip systems. The affected zones are subjected to an extra activity of three or four slip systems.

(3) The width and shape of the affected zones are not symmetrical with respect to GB. Crystal B experiences a stronger GB effect than Crystal A. The affected zone of Crystal B has a feathery appearance.

\section{Acknowledgements}

One of the authors (KK) thanks Dr. N. Hansen, Dr. D. J. Jensen and Dr. F. Inoko for scientific discussions. KK is also grateful to Dr. Okada for providing SEM facilities.

\section{REFERENCES}

1) J. D. Livingston and B. Chalmers: Acta Metall. 5 (1957) 322-327.

2) Z. Shen, R. H. Wagoner and W. A. T. Clark: Acta Metall. 36 (1988) 3231-3242.

3) T. C. Lee, I. M. Robertson and H. K. Birnbaum: Metal. Trans. 21A (1990) 2437-2447.

4) J. Hjelen, R. Orsund and E. Nes: Acta Metall. Mater. 39 (1991) 13771404.

5) D. Juul Jensen: Mater. Sci. Technol. 16 (2000) 1360-1363.

6) D. Raabe, M. Sachtleber, Z. Zhao, F. Roters and S. Zaefferer: Acta Mater. 49 (2001) 3433-3441.

7) A. Tatschl and O. Kolednik: Mater. Sci. Eng. A 364 (2004) 384-399.

8) C. Thorning, M. A. J. Somers and J. A. Wert: Mater. Sci. Eng. A 397 (2005) 215-228.

9) J. A. Wert, Q. Liu and N. Hansen: Acta Mater. 45 (1997) 2565-2576.

10) Q. Liu and N. Hansen: Proc. R. Soc. Lond. A 454 (1998) 2555-2591.

11) N. Tsuji, T. Shinmiya, Y. Saito and M. Muraki: ISIJ Inter. 38 (1998) 380-389.

12) Q. Liu, J. A. Wert and N. Hansen: Acta Mater. 48 (2000) 4267-4279.

13) F. Basson and J. H. Driver: Acta Mater. 48 (2000) 2101-2115.

14) T. Okada, L. Ikeda, X. Huang, J. A. Wert, K. Kashihara and F. Inoko: Mater. Trans. 42 (2001) 1938-1944.

15) M. Haberjahn, P. Klimanek and M. Motylenko: Mater. Sci. Eng. A 324 (2002) 196-199.

16) T. Okada, X. Huang, K. Kashihara, F. Inoko and J. A. Wert: Acta Mater. 51 (2003) 1827-1839.

17) J. A. Wert: Acta Mater. 50 (2002) 3125-3139.

18) J. A. Wert, X. Huang and F. Inoko: Proc. Roy. Soc. Lond. A 459 (2003) 85-108.

19) Z. J. Li, A. Godfrey and Q. Liu: Acta Mater. 52 (2004) 149-160.

20) J. A. Wert, K. Kashihara, T. Okada, X. Huang and F. Inoko: Philos. Mag. 85 (2005) 1989-2006.

21) S. Zaefferer, J. C. Kuo, Z. Zhao, M. Winning and D. Raabe: Acta Mater. 51 (2003) 4719-4735.

22) M. C. Theyssier and D. H. Driver: Mater. Sci. Eng. A 272 (1999) 7382.

23) H. R. Z. Sandim, J. F. C. Lins, A. L. Piano and A. F. Padilha: Mater. Sci. Eng. A 354 (2003) 217-228.

24) N. C. Krieger Lassen, D. Juul Jensen and K. Conradsen: Scanning Microsc. 6 (1992) 115-121.

25) J. F. Bishop and R. Hill: Philos. Mag. 42 (1951) 1298-1307.

26) J. J. Hauser and B. Chalmers: Acta Metall. 9 (1961) 802-818.

27) R. E. Hook and J. P. Hirth: Acta Metall. 15 (1967) 1099-1110.

28) A. Tatschl and O. Kolednik: Mater. Sci. Eng. A 339 (2003) 265-280. 